Eur Urol. 2015 November ; 68(5): 756-765. doi:10.1016/j.eururo.2015.03.020.

\title{
Predicting Life Expectancy in Men Diagnosed with Prostate Cancer
}

\author{
Jesse D. Sammon ${ }^{1}$, Firas Abdollah ${ }^{1}$, Anthony D'amico², Matthew Gettman ${ }^{3}$, Alexander \\ Haese $^{4}$, Nazareno Suardi ${ }^{5}$, Andrew Vickers ${ }^{6}$, and Quoc-Dien Trinh ${ }^{7}$ \\ ${ }^{1}$ VUI Center for Outcomes Research Analytics and Evaluation, Henry Ford Health System, \\ Detroit, MI, USA \\ 2Department of Radiation Oncology, Dana-Farber Cancer Institute, Brigham and Women's \\ Hospital, Harvard Medical School, Boston, MA, USA \\ ${ }^{3}$ Department of Urology, Mayo Clinic, Rochester, Minnesota 55905, USA \\ ${ }^{4}$ Department of Urology, University of Hamburg Eppendorf, Hamburg, Germany \\ ${ }^{5}$ Department of Urology, Urological Research Institute, University Vita-Salute San Raffaele, Milan, \\ Italy \\ ${ }^{6}$ Memorial Sloan Kettering Cancer Center, New York, NY, USA \\ ${ }^{7}$ Division of Urologic Surgery and Center for Surgery and Public Health, Brigham and Women's \\ Hospital / Dana-Farber Cancer Institute, Harvard Medical School, Boston, MA, USA
}

\begin{abstract}
Context-The widespread use of prostate specific antigen (PSA) screening, has lead to the detection of more indolent prostate cancer $(\mathrm{CaP})$ in healthy men. Prostate cancer treatment and screening must therefore balance the potential for life gained vs. the potential for harm.

Fundamental to this balance is physician awareness of a patient's estimated life expectancy.

Objective-To review the evidence on life expectancy (LE) differences between men diagnosed with $\mathrm{CaP}$ and the general population. To examine clinician and model predicted LE and examine publicly available LE calculators
\end{abstract}

Evidence Acquisition-A comprehensive search of the PubMed database between 1990 and September 2014 was performed according to Preferred Reporting Items for Systematic Reviews and Meta-analysis (PRISMA) statement guidelines. Free text protocols of the following search terms were used "life expectancy prostate cancer", "life expectancy non-cancer", "non-cancer mortality prostate", "comorbidity-adjusted life expectancy". Two internet search engines were queried daily, for one month, for the search term "life expectancy calculator", and the top 20 results were examined.

Corresponding author: Jesse D. Sammon, D.O., VUI Center for Outcomes Research Analytics and Evaluation, Henry Ford Health System, 2799 W. Grand Boulevard | Detroit, Michigan | USA | 48202, Phone: +1.207.692.7167, Fax: +1.313.916.4352, jsammon79@gmail.com; jsammon1@hfhs.org.

Conflict of interest disclosures: Firas Abdollah is a consultant for GenomeDx biosciences. 
Evidence Synthesis-Of 992 articles and 32 websites screened, 17 articles and 9 websites were selected for inclusion. Men with non-screening detected $\mathrm{CaP}$, as well as distant disease at diagnosis, were found to have a shorter LE than age-matched peers, whereas men with localized $\mathrm{CaP}$ had a prolonged LE. In general, clinician-predicted 10-yr LE was pessimistic and of limited accuracy, however model-predicted LE provided only modest improvements in accuracy (c-index of models: 0.65-0.84). Online LE calculators vary in ease of use, but government life tables provide LE estimates near the mean of all examined calculators.

Conclusion-The accuracy of clinician-predicted survival is limited and while available statistical models offer improvement in discrimination, it is unclear that they provide advantages over freely available government life-tables.

Patient Summary-In this report we examine the differences in life expectancy for men diagnosed with prostate cancer compared to the general population and ways of predicting life expectancy to help guide treatment decisions. We found that current models of predicting life expectancy, specific to prostate cancer, might not be any better than government life tables or simple rules-of-thumb.

\section{Keywords}

Prostate cancer; Life Expectancy; Survival; Prediction

\section{Introduction}

Following the adoption of widespread prostate specific antigen (PSA) screening, cases of prostate cancer $(\mathrm{CaP})$ are largely screening detected. This has had profound effects on both the incidence and prevalence of $\mathrm{CaP}[1,2]$ and has introduced biases, associated with screening detected cancer, that have made the assessment of competing mortality central to the care of patients with $\mathrm{CaP}[3]$. These include selection bias, lead-time bias and length bias.

In 2012, only $40 \%$ of the U.S. male population over 50 indicated having undergone PSA testing in the previous year [4]. Not all men offered screening will undertake it, and the population that does may differ significantly from the broader at risk population (figure 1a). On one hand, those who consider themselves at higher risk (AAM, positive family history) may elect to undergo screening, however individuals who undergo screening have better overall health behaviors than those who do not. Patients electing to undergo screening may have better diets and healthier lifestyles and may more often choose definitive treatment when diagnosed with cancer[2]. The 'healthy screener' effect was demonstrated in a study of women self-referred for ovarian cancer screening; the rate of mortality from ovarian cancer in the screened cohort was similar to the expected rate of mortality in the unscreened population. Nonetheless, the screened population had lower than expected rates of mortality from colorectal and lung cancer, suggesting healthier life choices and diet as well as increased healthcare utilization[5].

While selection bias impacts the population diagnosed with $\mathrm{CaP}$, lead-time bias and length bias have profound effects on the characteristics of the cancer that is detected. Lead-time bias (Figure 1b) is demonstrated when a man undergoes PSA screening and has his prostate 
cancer detected $n$-years earlier than it otherwise would have been, he will live $n$-years longer with $\mathrm{CaP}$. Because prostate cancer is an indolent disease with a protracted preclinical state, the magnitude of this effect can be quite large; estimated to be as high as 12.3 years[6]. Length bias (Figure 1c) also occurs when cancers become detectable in their preclinical state. Rapidly fatal cancers remain in a pre-clinical state for a shorter period of time, limiting the utility of screening, whereas indolent cancers are detectable in the pre-clinical (asymptomatic) state for a prolonged period.

The net effect of these important biases has been increased detection of cancers that are more indolent in healthier people. On this basis, and the associated risk of overtreatment $[6$, 7], current guidelines recommend the use of life expectancy estimation to help balance the potential for life gained vs. potential for harm caused by diagnosis and/or treatment of $\mathrm{CaP}$. [3] The purpose of this review is two-fold. We first review studies examining the difference in life expectancy between men diagnosed with $\mathrm{CaP}$ and the general population. We then review methods of predicting LE. We review studies of healthcare provider LE estimation, studies of model-predicted life expectancy, and finally publicly available, life expectancy calculators.

\section{Evidence Acquisition}

We conducted a systematic review of the PubMed database between 1990 and September 2014, according to the Preferred Reporting Items for Systematic Reviews and Meta-analysis statement guidelines.[8]

The predefined search terms "(((life expectancy prostate cancer) OR life expectancy noncancer) OR non-cancer mortality prostate) OR comorbidity adjusted life expectancy" was used to find 986 articles, which were screened for inclusion. A further 6 significant studies were identified by bibliography review. All experimental and observational study designs were eligible for inclusion in this review, including but not limited to controlled clinical trials, statistical modeling, case series, case-control, and cohort studies. Comments, editorials, and review articles were not considered eligible for inclusion. Seventeen studies were included in the review. The flow chart of the systematic literature review is Figure 2.

Predefined criteria for exclusion included: Non-English Language, assessment of treatment effect, articles that did not pertain to cancer, for studies reporting from the same dataset the most recent report was used. Two authors (J.S. and F.A.) separately reviewed the records to select the studies, with any discrepancy resolved the senior author's (Q-D.T.) determination of adherence to the pre-specified inclusion/exclusion criteria. Study type was ascertained and quality of evidence described. There was no examination of an intervention in any of the examined studies, therefore the pooling of outcome measures and meta-analysis were not undertaken.

The assessment of freely available LE calculators was made through Internet search. Two widely used search engines (Google.com, Bing.com) were queried for the keywords "life expectancy calculator". To account for variability in results among searches, the top 20 results were assessed daily for one month (July 15th-Aug 15th 2014). Websites that were 
duplicates of common actuarial data (ie. SSA life tables) or those lacking clear identifying of source data were excluded. Nine readily accessible online LE calculators were identified.

\section{Evidence Synthesis}

\section{Life Expectancy in men diagnosed with CaP (Table 1)}

Studies examining the effect of cancer on patient mortality have largely focused on the excess mortality attributable to cancer[9] and there is limited data on the non-cancer mortality of cancer patients. As the population of patients surviving cancer expands due to improved detection and treatment, the impact of other-cause mortality will become an increasingly pressing health policy concern. Understanding other-cause mortality requires knowledge of the specific cause of death (COD), the ascertainment of which has historically been very challenging to ascertain with a degree of accuracy sufficient for analysis.[10,11]

The most common approach to estimating the excess mortality associated with cancer has been relative survival, a method that does not rely on COD information.[12] Relative survival is the ratio of observed survival in a population of cancer patients relative to the background survival rate. Brenner and Arndt used this methodology to examine 180,605 men diagnosed with $\mathrm{CaP}$ in the U.S. between 1990 and 2000. In their study, 5- and 10-yr estimates of relative survival were $98.9 \%$ and $94.8 \%$, which indicates that excess mortality due to prostate cancer was as low as $1 \%$ and $5 \%$ within 5 and 10 years following diagnosis, respectively.[13] Interestingly, they noted an absence of excess mortality in men with wellor moderately-differentiated locoregional $\mathrm{CaP}$ across all age groups. For grades 1 and 2, the 5 -yr relative survival was 5-6\% higher in the CaP population than in the general population, while the 10-yr relative survival was 3-6\% higher. Cho et al confirmed these findings in a study of 2000-2006 U.S. registry data, using a left-truncated survival method with the hazard of death due to other causes characterized as a function of age. This study took advantage of a newly created algorithm to accurately ascribe COD in the Surveillance, Epidemiology, and End Results (SEER) program of the National Cancer Institute.[14] Looking directly at other cause survival, and comparing it to U.S. life tables, they found that patients with loco-regional $\mathrm{CaP}$ have a survival benefit over the baseline population ranging from 4 years in 70-year-olds to 6 years in 50-year-olds. This is in contrast to men diagnosed with distant disease, whose other-cause survival was lower than in the general population. [15] (Figure 3)

These studies present evidence of the effect of healthy screener bias in the context of PSA screening. Patients diagnosed at an early stage through PSA screening may have better access to healthcare, higher socioeconomic status and healthier behaviors, leading to a lower risk of other-cause mortality relative to the general population. Conversely, patients diagnosed with advanced cancer, who did not undergo routine PSA screening, may also ignore early CaP symptoms and have poor access to health care.[15] Data from the Queensland Cancer Registry support this premise. Examining other-cause mortality in a largely unscreened population of men with CaP diagnosed between 1982-2002, Baade et al found a mortality ratio of $32.6 \%$ higher than the general population, with increased mortality rates for poisoning, respiratory disease, digestive disease and cardiovascular disease.[16] 


\section{Clinician Predicted Life Expectancy Estimation (Table 2)}

PSA screening for the early detection of prostate cancer is a hotly contested and widely scrutinized practice. Currently, the US Preventative Services Task Force recommends against PSA screening in any age group.[17] Prior statements and guidelines from other national/international panels recommended screening only in men with a > 10 yr LE.[18-20] Nonetheless, as recently as 2012, the prevalence of screening remains higher in men over 80 (35.3\%) than men 55-69 (32.3\%).[4] Such data suggest that selective screening based on life expectancy is not routinely practiced. In addition to screening recommendations, guidelines for the treatment of $\mathrm{CaP}$ have also used the 10-yr threshold for recommending definitive treatment with RP or radiotherapy (RT).[21] Yet the premise underlying these recommendations was that healthcare providers have the ability to predict 10-yr LE. There is limited evidence to support this conjecture.

The first group to examine the "10-yr rule", Koch et al., examined the actuarial predicted life expectancy of 261 consecutive men undergoing RRP, based on the assumption that men undergoing active treatment were considered by the treating provider to have a LE greater than 10 years. In this retrospective review, they found that $20 \%$ of men undergoing RRP had a predicted LE less than 10 years.[22] While these estimates suggest that clinicians can correctly predict LE at 10 years $80 \%$ of the time, the effect of case mix plays a significant role. If the preponderance of patients were young and healthy, LE beyond 10 years is not in question. Similar findings were observed by Krahn et al., who examined the predictions of 191 urologists, reviewing 18 patient scenarios, and compared them to a Markov model predicting LE based on age and comorbidity. They found that urologists were able to accurately estimate $10-\mathrm{yr}$ LE in $82 \%$ of the scenarios examined.[23]

Wilson et al found that "even with detailed data on comorbidity, the clinicians in (their) study were generally inaccurate, imprecise and inconsistent in their predictions of patient 10-year survival"[24]. They came to this conclusion after comparing physician $(\mathrm{n}=18)$ reviews to actuarial assessments of survival. Interestingly, they described an inclination toward the underestimation of LE, with a mean underestimation of $10.8 \%$. In a hypothetical situation where guidelines suggest treatment if the estimated 10 -yr survival is $>50 \%$, the authors determined physicians would on average recommend treatment $66 \%$ of the time in patients with $>10$-yr estimated LE, denying $36 \%$ of men appropriate treatment. Inappropriate treatment for men with $\mathrm{LE}<10$ years was only $24 \%$.

A limitation of the above studies was the absence of real patient survival data and the comparison to modeled survival estimates (both the Markov model and actuarial estimates have their own limitations), thus limiting the utility of the study findings. Two groups overcame this limitation by comparing clinician-predicted survival (CPS) to actual survival (AS). Walz et al. examined the accuracy of 19 clinicians in predicting 10-yr survival of 50 patients undergoing EBRT or RP (40\% had other cause mortality). Examination of the area under the curve (AUC) for receiver operating characteristic curves found that attending urologists had a discrimination of $0.67(0.60-0.72)$, residents 0.69 (0.64-0.74) and medical students 0.67 (0.58-0.76). The absence of meaningful difference between these groups suggests "that neither expertise nor exposure time are important in predicting LE"[25]; both the best and worst predictions were made by urology staff. Leung et al found nearly 
identical findings in an analysis of 100 clinicians reviewing seven clinical scenarios summarized from the charts of deceased patients. They found that the mean estimated LE was -2 yrs (SD: $6.1 \mathrm{yr}$ ). When the authors dichotomized estimates in terms of correctly identifying which patients would live more than or less than 10 years, physicians were correct $68 \%$ of the time. They found that residents were more accurate than students or attending urologists, and found internal medicine clinicians more accurate than either Urologists or primary care providers.

A common theme amongst articles comparing the ability of healthcare providers to predict LE is that inaccuracies in prediction tend to be unrelated to level of training or experience. The problem lies in the fact that "inconsistent predictions of LE may lead to patients being managed differently by the same or different doctors despite identical comorbidity".[26] A second common theme is that healthcare provider LE estimates tend to be overly optimistic when considering patients with short LE, as in patients with metastatic disease presenting for palliative treatment[27, 28]; whereas estimates of 10-yr LE are largely pessimistic. $[23-25,29]$

\section{Model-Predicted LE (Table 3)}

The prediction of LE is challenging and empiric predictions of LE are subject to an individual clinician's experience, judgment, underlying personal beliefs and prejudices. Furthermore, the accurate prediction of LE of patients with prostate cancer is particularly challenging as patients with prostate cancer in the post-PSA era are largely diagnosed through screening and this population tends to live longer than their non-cancer peers (healthy screener bias, Figure 1). As a consequence of the inherent limitations in clinicianpredicted survival, several investigators have sought to model overall survival in men undergoing treatment for $\mathrm{CaP}$.

Albertsen et al examined a cohort of men diagnosed with CaP between 1971-1976 from the Connecticut cancer registry $(n=451)$. The goal of their study was to examine the effect of comorbidity on LE. As such, they examined the effect of three comorbidity indexes on survival (Kaplan-Feinstein index, Charlson Comorbidity Index, Index of Coexisting Disease). This study was novel in that it clearly demonstrated the importance of comorbidity in LE predictions; in a external validation of the model, it was found to have a c-index of $0.71 .[30,31]$ Tewari et al developed a series of lookup tables incorporating several clinical and demographic characteristics: age, race, comorbidity, prostate specific antigen, cancer grade, and treatment type.[32] The discrimination of this model was 0.69 for overall survival and 0.63 for cancer specific survival. In external validation, discrimination was found to be 0.70.[30]

Cowen et al performed the aforementioned external validations on a retrospective cohort of men receiving treatment for $\mathrm{CaP}$ and followed for 13 years (1989-2002). They also created a nomogram incorporating numerous predictors including age, CCI, performance status, angina history, blood pressure, body mass index, tobacco use, marital status, PSA, Gleason sum, clinical stage, treatment type and treatment year. The discrimination of their 10-yr survival model predictions was 0.73 , a modest improvement over the Albertsen and Tewari models. 
The model with the highest c-index published to date is by Walz et al. examining the risk of non-cancer mortality within 10 years of receiving definitive therapy.[33] The population used to design and test their model included 9131 Canadian men treated with either radical prostatectomy $(n=5955)$ or EBRT $(n=3176)$ between 1989 and 2000 and excluding all men who died from CaP. Incorporating age, treatment type and CCI, the model discrimination was 0.84 . While the approach of including treatment selection into the model is appealing, as it incorporates any presumed survival advantage associated with RP, its inclusion also incorporates the biases associated with treatment selection directly into the model. In general $\mathrm{RP}$ is offered to healthier men then RT, so it is unsurprising that they live longer, and incorporation of this variable improves model discrimination.

Finally, Marriotta et al have recently developed a novel approach to race- and genderspecific life tables.[34] Using SEER-Medicare patients between 1992-2005 ( $\mathrm{n}=1,108,085)$, the effect of individual comorbidities on survival was estimated with Cox proportional hazards modeling; the coefficient estimates of the condition indicators then comprised the weights for each comorbid condition. For each patient, an individual comorbidity score was calculated as a sum of these weights. From these weights, an individual health- and ageadjusted LE were calculated. There are limitations to this approach, as the life tables were created for all cancers and are not specific for CaP. Furthermore, the discrimination of the models is modest for younger patients soon after diagnosis ( $66 \mathrm{yr}$-old women $=0.73,66 \mathrm{yr}$ old man $=0.68$ ), but is poor in older patients 10 years after diagnosis $(80 \mathrm{yr}$-old women $=$ $0.58,80 \mathrm{yr}$-old man $=0.57$ ). Nonetheless their approach has the advantage of being able to be incorporated into the analysis of observational data.[35]

\section{Actuarial Predicted Survival (Table 4)}

Given that actuarial predicted survival is considered as the standard for LE prediction by several of the previous studies, we assessed the calculators freely available to the public and ascertained their usability in clinical practice. Within the top 20 web hits for the search term "life expectancy calculator", 9 unique LE prediction tools were found (see Table 4).[36-44] The number of data points to calculate LE ranged from 2 (Social Security Administration) to 40 (Livingto100.com).[37, 44] The most common questions asked (in over 75\% of calculators) were gender, age, weight, height, smoking, alcohol intake, driving habits, blood pressure. Only a single calculator (Wharton/UPENN) asked about prostate cancer history. [39]

To estimate the variance in calculator-predicted LE, we calculated the LE in each of the 9 calculators for a series of hypothetical patient. For example: a 65yo, white male, 68 inches tall, $185 \mathrm{lbs}$, hypercholesterolemia on statins, no family history, 45 pack-yr smoking history, married, non-manual labor, $\$ 50,000$ annual income, normal sleep patterns. SSA life table predictions demonstrated a LE of 83.9 yrs for all white men aged 65, the range of actuarial predictions for the hypothetical patient was from 77.5 (Bankrate) to 84.88 (Wharton).[39, 43]

The fact that SSA life tables provide estimates of LE near the mean for the freely available calculators (82.66yrs(SD +/- 3.44)) is of clinical importance as LE estimates from SSA life tables can be easily ascertained and incorporated into patient counseling. While the 
methodology of online calculators is proprietary and not published alongside the calculators, they appear to provide fairly consistent estimates of LE. This is unsurprising given that most ask similar questions about the major risk factors for cardiovascular disease, the leading cause of death in the US. Given that many men undergoing treatment for $\mathrm{CaP}$ receive androgen deprivation therapy (which is associated with both fatal and non-fatal CV and metabolic events[45]) as a component of their treatment, LE estimates incorporating CV risk factors may require further adjustment.

\section{Discussion}

To summarize, the population of men diagnosed with $\mathrm{CaP}$ is different from similarly aged men without $\mathrm{CaP}$ diagnosis, in large part due to selection bias and the "healthy screener" effect. Two important biases associated with screening diagnosed cancers, lead-time and length bias, suggest that contemporary patients diagnosed with $\mathrm{CaP}$ will live for a significant period of time with the diagnosis. This is supported by data showing that the LE for most $\mathrm{CaP}$ patients is in fact higher than what would be expected in age- and race-matched peers[15] and that diagnosis with loco-regional $\mathrm{CaP}$ is not associated with excess mortality. [13]

Consequently, the accurate estimation of LE in CaP patients is of paramount importance to avoid unnecessary treatment in men unlikely to benefit, but also to afford men with a protracted LE the opportunity for cure. While on average, avoiding surgery in men over 70 and radiotherapy in men over 75 unless cancer is very aggressive and patient is above average health may be prudent, personalizing this decision based on an individuals LE using age and comorbidity would be ideal.

The ability of the currently available prediction models to discriminate 10-yr LE ranged from 0.65-0.84, however discrimination (C-index, AUC), as a measure of a predictive model's performance, has significant limitations. Discrimination measures how well a model can discriminate between two hypothetical patients (ie. which patient has a higher vs. lower LE or higher vs. lower probability of 10-yr LE). It is critically dependent on the variation of predictors in the modeled cohort as well as the prevalence of the predictor in the cohort. Calibration is the characteristic of a model that describes how close a prediction is to an actual event. Studies of the available models emphasize discrimination but model calibration is not discussed in depth. Furthermore, a model may be well calibrated on one dataset and poorly calibrated on another. For a predictive model to be of clinical use its performance on a population of interest must first be ascertained[46].

\section{Conclusion}

The accuracy of clinician-predicted survival is limited and it is unclear that the available statistical models provide any advantages over freely available government life-tables. The question of how life expectancy should be estimated remains unanswered. 


\section{Acknowledgments}

The authors would like to thank Akshay Sood, M.D. for his invaluable assistance with the exploration of internetbased life-expectancy calculators.

Quoc-Dien Trinh is supported by the Professor Walter Morris-Hale Distinguished Chair in Urologic Oncology at Brigham and Women's Hospital.

\section{References}

1. Potosky AL, Feuer EJ, Levin DL. Impact of screening on incidence and mortality of prostate cancer in the United States. Epidemiologic reviews. 2001; 23(1):181-6. [PubMed: 11588846]

2. Kramer BS. The science of early detection. Urol Oncol. 2004; 22(4):344-7. [PubMed: 15283894]

3. Mohler JL, Kantoff PW, Armstrong AJ, et al. Prostate cancer, version 2.2014. Journal of the National Comprehensive Cancer Network : JNCCN. 2014; 12(5):686-718. [PubMed: 24812137]

4. Sammon JD, Pucheril D, Diaz M, et al. Contemporary Nationwide Patterns of Self-reported Prostate-Specific Antigen Screening. JAMA internal medicine. 2014; 174(11):1839-41. [PubMed: 25179266]

5. Crayford TJ, Campbell S, Bourne TH, Rawson HJ, Collins WP. Benign ovarian cysts and ovarian cancer: a cohort study with implications for screening. Lancet. 2000; 355(9209):1060-3. [PubMed: 10744092]

6. Draisma G, Boer R, Otto SJ, et al. Lead times and overdetection due to prostate-specific antigen screening: estimates from the European Randomized Study of Screening for Prostate Cancer. J Natl Cancer Inst. 2003; 95(12):868-78. [PubMed: 12813170]

7. Loeb S, Bjurlin MA, Nicholson J, et al. Overdiagnosis and overtreatment of prostate cancer. Eur Urol. 2014; 65(6):1046-55. [PubMed: 24439788]

8. Liberati A, Altman DG, Tetzlaff J, et al. The PRISMA statement for reporting systematic reviews and meta-analyses of studies that evaluate health care interventions: explanation and elaboration. PLoS medicine. 2009; 6(7):e1000100. [PubMed: 19621070]

9. Coleman MP, Quaresma M, Berrino F, et al. Cancer survival in five continents: a worldwide population-based study (CONCORD). Lancet Oncol. 2008; 9(8):730-56. [PubMed: 18639491]

10. Begg CB, Schrag D. Attribution of deaths following cancer treatment. Journal of the National Cancer Institute. 2002; 94(14):1044-5. [PubMed: 12122090]

11. Percy C, Stanek E 3rd, Gloeckler L. Accuracy of cancer death certificates and its effect on cancer mortality statistics. Am J Public Health. 1981; 71(3):242-50. [PubMed: 7468855]

12. Ederer F, Axtell LM, Cutler SJ. The relative survival rate: a statistical methodology. National Cancer Institute monograph. 1961; 6:101-21. [PubMed: 13889176]

13. Brenner H, Arndt V. Long-term survival rates of patients with prostate cancer in the prostatespecific antigen screening era: population-based estimates for the year 2000 by period analysis. $\mathrm{J}$ Clin Oncol. 2005; 23(3):441-7. Epub 2004/12/02. [PubMed: 15572727]

14. Howlader N, Ries LA, Mariotto AB, Reichman ME, Ruhl J, Cronin KA. Improved estimates of cancer-specific survival rates from population-based data. J Natl Cancer Inst. 2010; 102(20):158498. [PubMed: 20937991]

15. Cho H, Mariotto AB, Mann BS, Klabunde CN, Feuer EJ. Assessing non-cancer-related health status of US cancer patients: other-cause survival and comorbidity prevalence. Am J Epidemiol. 2013; 178(3):339-49. Epub 2013/07/05. [PubMed: 23825168]

16. Baade PD, Fritschi L, Eakin EG. Non-cancer mortality among people diagnosed with cancer (Australia). Cancer Causes Control. 2006; 17(3):287-97. Epub 2006/02/21. [PubMed: 16489536]

17. Moyer VA. Screening for prostate cancer: U.S. Preventive Services Task Force recommendation statement. Ann Intern Med. 2012; 157(2):120-34. Epub 2012/07/18. [PubMed: 22801674]

18. Wolf AM, Wender RC, Etzioni RB, et al. American Cancer Society guideline for the early detection of prostate cancer: update 2010. CA Cancer J Clin. 2010; 60(2):70-98. [PubMed: 20200110]

Eur Urol. Author manuscript; available in PMC 2015 November 01. 
19. Mistry S, Mayer W, Khavari R, Ayala G, Miles B. Can Urol Assoc J. 2009; 3(3):205-10. Epub 2009/06/23. Who's too old to screen? Prostate cancer in elderly men. [PubMed: 19543463]

20. Greene KL, Albertsen PC, Babaian RJ, et al. Prostate specific antigen best practice statement: 2009 update. J Urol. 2009; 182(5):2232-41. Epub 2009/09/29. [PubMed: 19781717]

21. Mohler JL. The 2010 NCCN clinical practice guidelines in oncology on prostate cancer. Journal of the National Comprehensive Cancer Network : JNCCN. 2010; 8(2):145. [PubMed: 20141674]

22. Koch MO, Miller DA, Butler R, Lebos L, Collings D, Smith JA Jr. Are we selecting the right patients for treatment of localized prostate cancer? Results of an actuarial analysis. Urology. 1998; 51(2):197-202. Epub 1998/03/12. [PubMed: 9495697]

23. Krahn MD, Bremner KE, Asaria J, et al. The ten-year rule revisited: accuracy of clinicians' estimates of life expectancy in patients with localized prostate cancer. Urology. 2002; 60(2):25863. Epub 2002/07/26. [PubMed: 12137823]

24. Wilson JR, Clarke MG, Ewings P, Graham JD, MacDonagh R. The assessment of patient lifeexpectancy: how accurate are urologists and oncologists? BJU Int. 2005; 95(6):794-8. Epub 2005/03/30. [PubMed: 15794785]

25. Walz J, Gallina A, Perrotte P, et al. Clinicians are poor raters of life-expectancy before radical prostatectomy or definitive radiotherapy for localized prostate cancer. BJU Int. 2007; 100(6): 1254-8. Epub 2007/11/06. [PubMed: 17979925]

26. Clarke MG, Ewings P, Hanna T, Dunn L, Girling T, Widdison AL. How accurate are doctors, nurses and medical students at predicting life expectancy? European journal of internal medicine. 2009; 20(6):640-4. Epub 2009/09/29. [PubMed: 19782929]

27. Muers MF, Shevlin P, Brown J. Prognosis in lung cancer: physicians' opinions compared with outcome and a predictive model. Thorax. 1996; 51(9):894-902. [PubMed: 8984699]

28. Chow E, Davis L, Panzarella T, et al. Accuracy of survival prediction by palliative radiation oncologists. Int J Radiat Oncol Biol Phys. 2005; 61(3):870-3. Epub 2005/02/15. [PubMed: 15708268]

29. Leung KM, Hopman WM, Kawakami J. Challenging the 10-year rule: The accuracy of patient life expectancy predictions by physicians in relation to prostate cancer management. Can Urol Assoc J. 2012; 6(5):367-73. Epub 2012/10/25. [PubMed: 23093629]

30. Cowen ME, Halasyamani LK, Kattan MW. Predicting life expectancy in men with clinically localized prostate cancer. J Urol. 2006; 175(1):99-103. Epub 2006/01/13. [PubMed: 16406881]

31. Albertsen PC, Fryback DG, Storer BE, Kolon TF, Fine J. The impact of co-morbidity on life expectancy among men with localized prostate cancer. J Urol. 1996; 156(1):127-32. Epub 1996/07/01. [PubMed: 8648773]

32. Tewari A, Johnson CC, Divine G, et al. Long-term survival probability in men with clinically localized prostate cancer: a case-control, propensity modeling study stratified by race, age, treatment and comorbidities. J Urol. 2004; 171(4):1513-9. Epub 2004/03/17. [PubMed: 15017210]

33. Walz J, Gallina A, Saad F, et al. A nomogram predicting 10-year life expectancy in candidates for radical prostatectomy or radiotherapy for prostate cancer. J Clin Oncol. 2007; 25(24):3576-81. Epub 2007/08/21. [PubMed: 17704404]

34. Mariotto AB, Wang Z, Klabunde CN, Cho H, Das B, Feuer EJ. Life tables adjusted for comorbidity more accurately estimate noncancer survival for recently diagnosed cancer patients. $\mathrm{J}$ Clin Epidemiol. 2013; 66(12):1376-85. Epub 2013/09/17. [PubMed: 24035494]

35. Sammon JD, Abdollah F, Reznor G, et al. Patterns of Declining Use and the Adverse Effect of Primary Androgen Deprivation on All-cause Mortality in Elderly Men with Prostate Cancer. Eur Urol. 2014

36. System, MSR. [Access Date:8/15/2014] Life Expectancy Calculator. http://www.msrs.state.mn.us/ info/Age_Cal.htmls

37. Perls, T. [Access Date:8/15/2014] The Living to 100 Life Expectancy Calculator. https://http:// www.livingto100.com/calculator

38. Mutual, N. [Access Date:8/15/2014] Lifespan Calculator. http://media.nmfn.com/tnetwork/ lifespan/

39. Foster, D.; Chua, CT.; Ungar, LH. [Access Date:8/15/2014] Wharton/UPENN Life Calculator. http://gosset.wharton.upenn.edu/mortality/perl/CalcForm.html 
40. Financial, G. [Access Date:8/16/2014] Genworth Financial Companies Calculator. https:// pro.genworth.com/amm/public_contents/calculators/8830.html

41. Company, MI. [Access Date:8/15/2014] Longevity Calculator. https://rslic.metlife.com/lic/ corpLongevity.do

42. Company, JHI. [Access Date:8/15/2014] https://http://www.johnhancockinsurance.com/life/lifeexpectancy-tool.aspx

43. Bankratre.com. [Access Date:8/15/2014] Life Expectancy Calculator. http://www.bankrate.com/ calculators/retirement/life-age-expectancy-calculator.aspx

44. Administration, SS. [Access Date:8/15/2014] Retirement \& Survivors Benefits: Life Expectancy Calculator. http://www.socialsecurity.gov/oact/population/longevity.html

45. D'Amico AV, Denham JW, Crook J, et al. Influence of androgen suppression therapy for prostate cancer on the frequency and timing of fatal myocardial infarctions. J Clin Oncol. 2007; 25(17): 2420-5. [PubMed: 17557956]

46. Vickers AJ, Cronin AM. Everything you always wanted to know about evaluating prediction models (but were too afraid to ask). Urology. 2010; 76(6):1298-301. [PubMed: 21030068] 
$1 a$
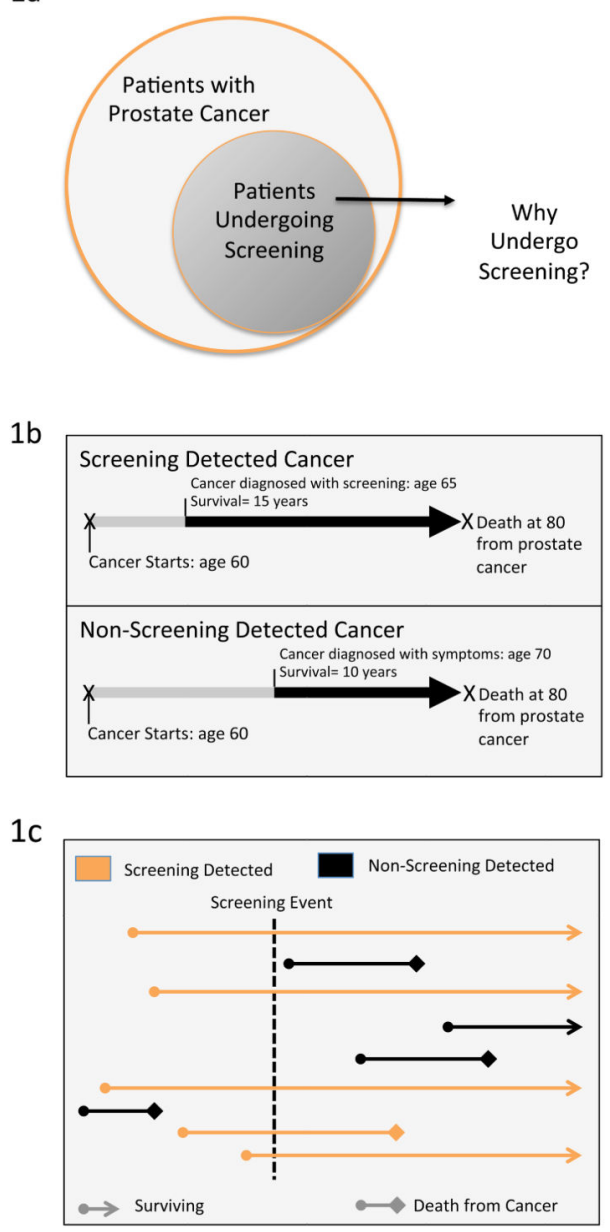

Figure 1.

Three biases associated with screening detected cancers that necessitate the assessment of competing mortality when treating men with prostate cancer $(\mathrm{CaP})$. Selection bias or the "healthy screener effect" (Figure 1a): Men undergoing prostate specific antigen (PSA) screening may have better diets, healthier lifestyles, and improved access to care relative to unscreened men. Lead-time bias (Figure 1b): the detection of prostate cancer with PSA can lead to improved perceived survival in absence of any modification of the disease course.

Length bias (Figure 1c): PSA screening detects $\mathrm{CaP}$ in the asymptomatic phase; because fast-growing tumors have a shorter asymptomatic phase than slower-growing tumors, PSA screening will preferentially detect indolent cancer. 


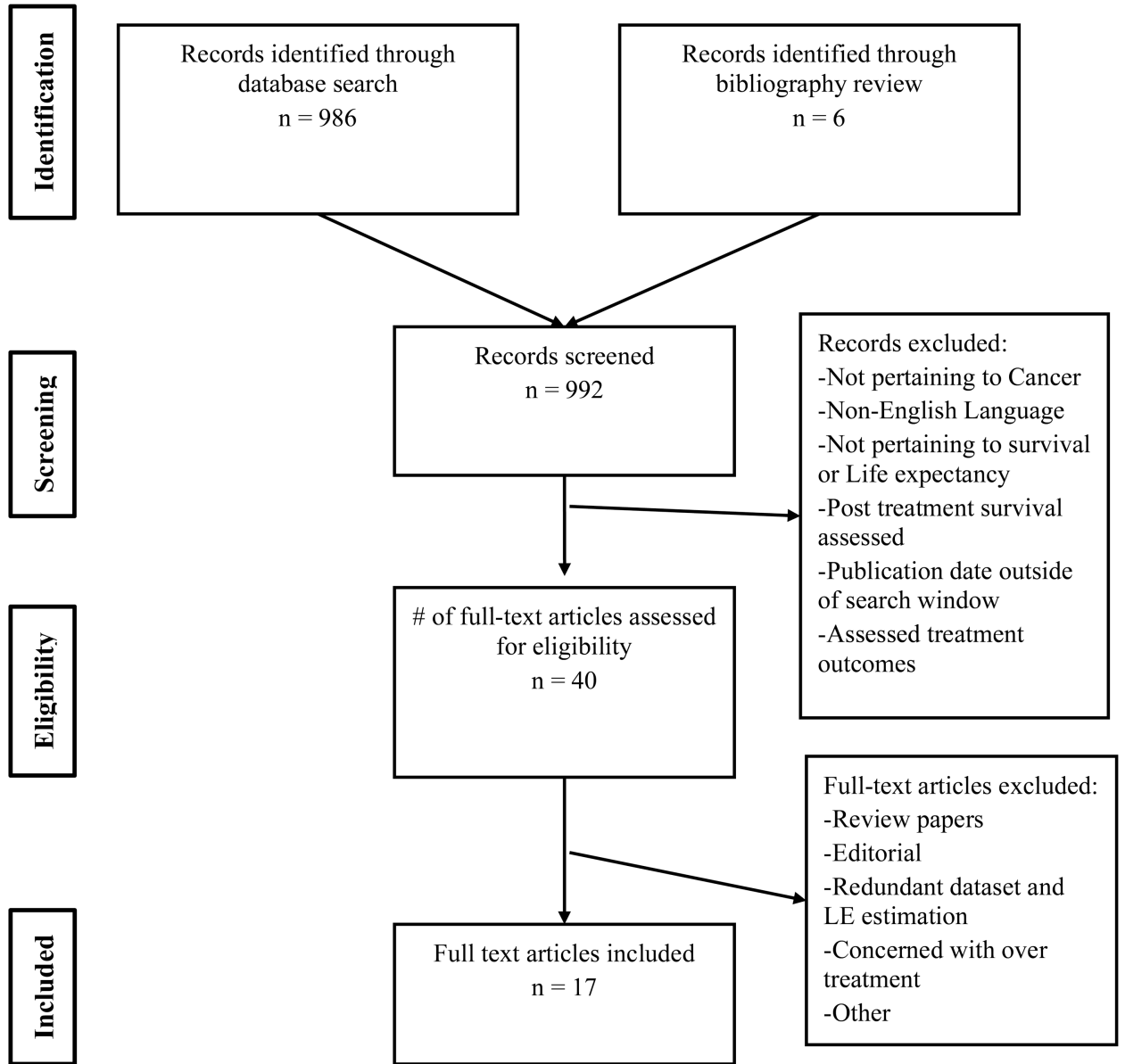

Figure 2.

Flow chart of the systematic review. 

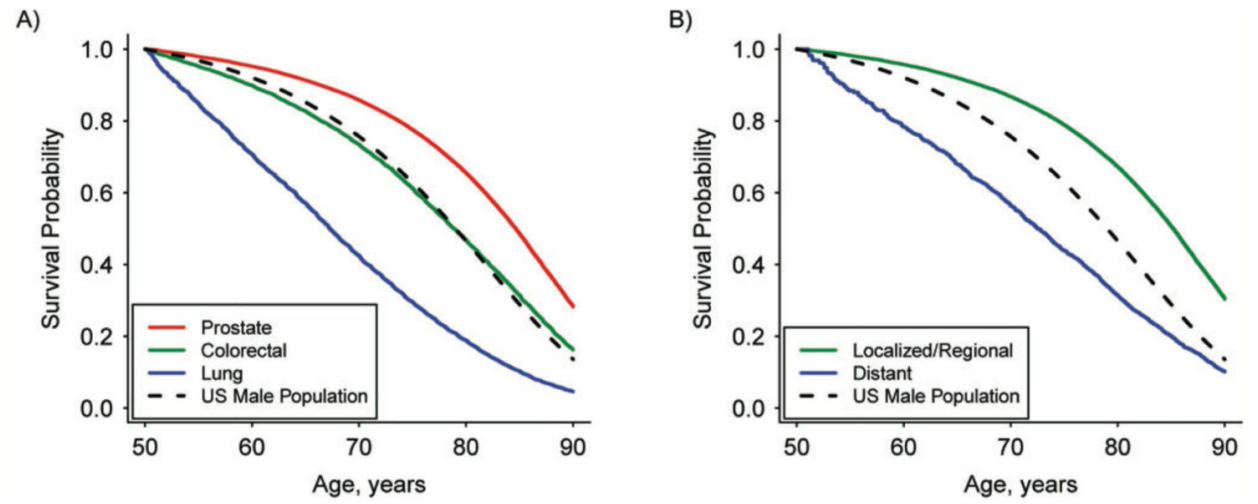

Figure 3.

The "healthy screener" effect in CaP: Compared to U.S. life tables, patients with locoregional prostate cancer have an other-cause survival benefit over the baseline population ranging from 4 years in 70-year-olds to 6 years in 50-year-olds. This is in contrast to men diagnosed with distant disease, whose other-cause survival was lower than in the general population. Estimated non-cancer survival probability for male cancer patients conditional on surviving to 50 years of age, Surveillance, Epidemiology, and End Results, 2000-2006. Reproduced with permission, from Cho et al American Journal of Epidemiology, Oxford University Press. 


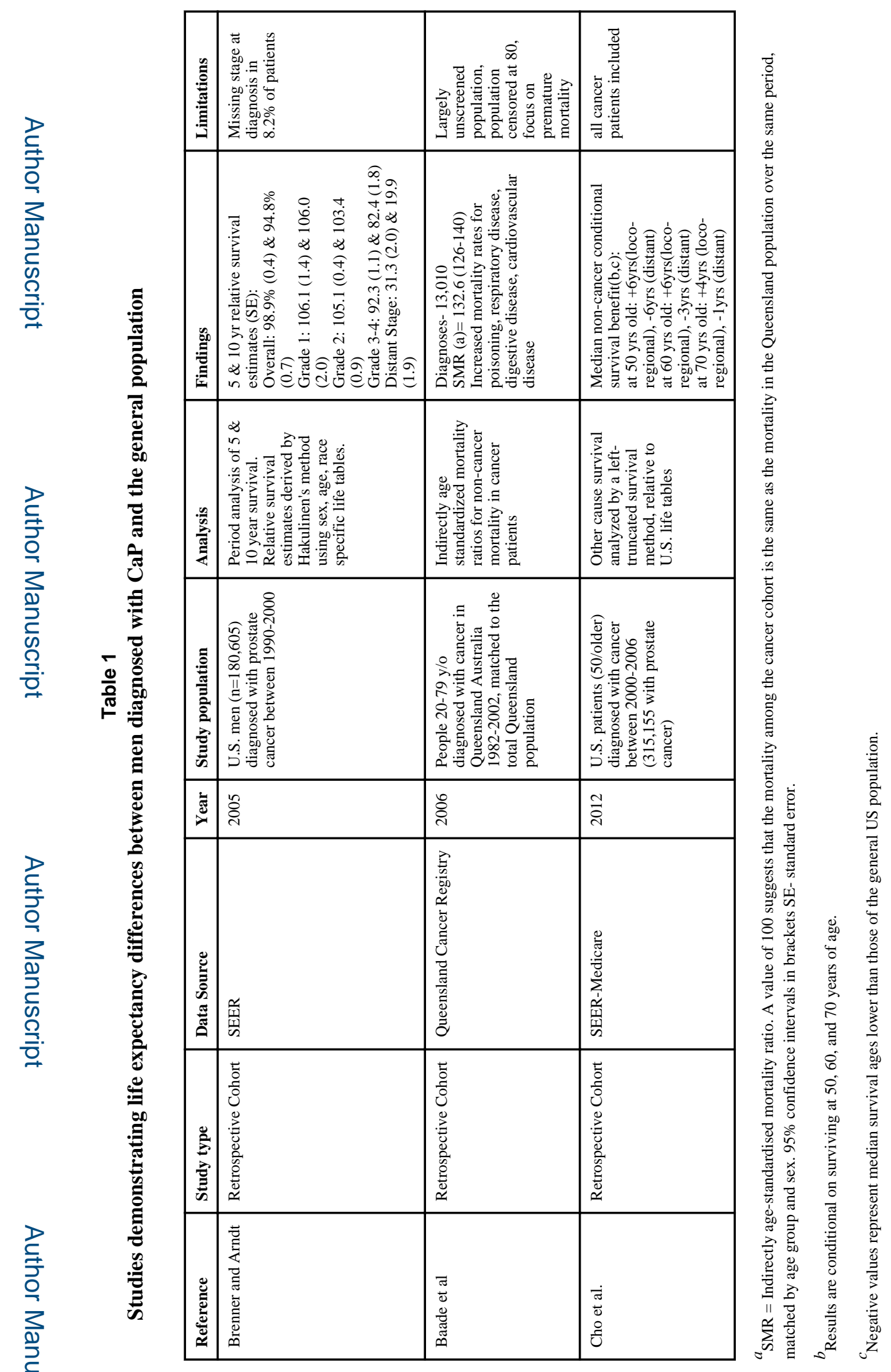

Eur Urol. Author manuscript; available in PMC 2015 November 01. 


\begin{tabular}{|c|c|c|c|c|c|c|}
\hline 象 & 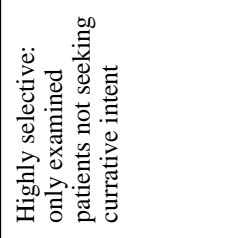 & 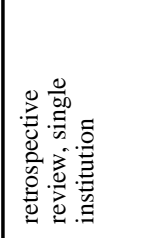 & 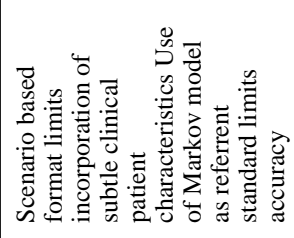 & 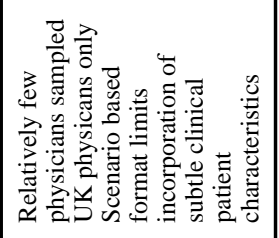 & 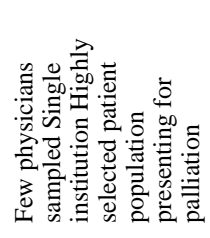 & 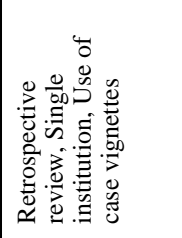 \\
\hline 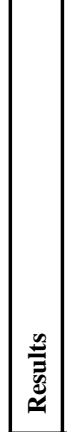 & 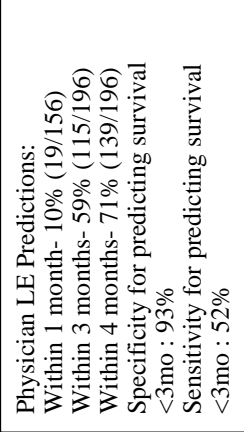 & 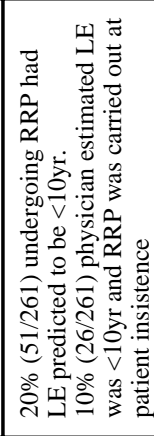 & 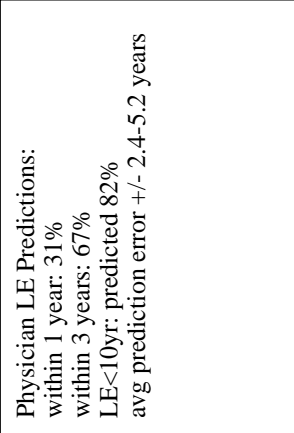 & 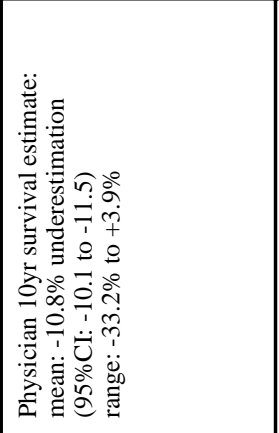 & 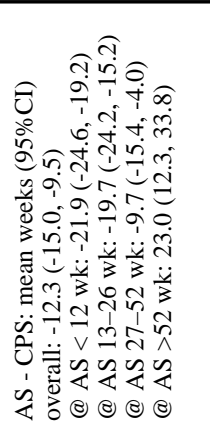 & 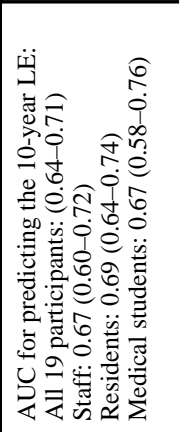 \\
\hline 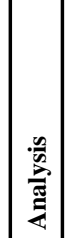 & 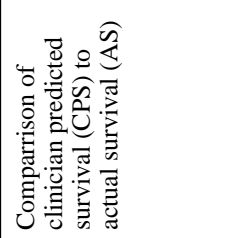 & 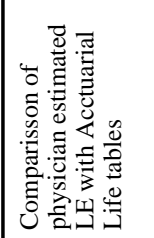 & 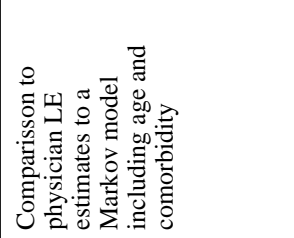 & 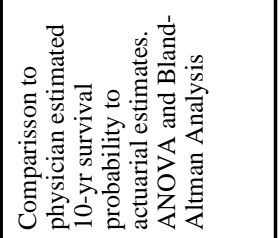 & 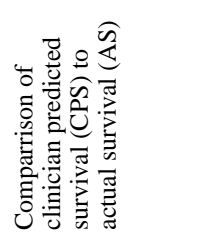 & 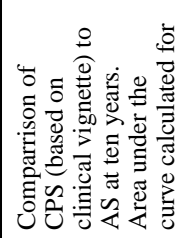 \\
\hline 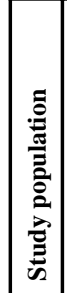 & 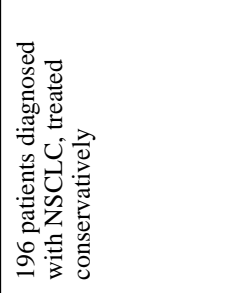 & 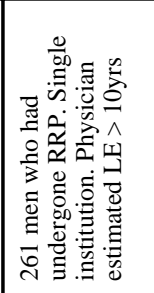 & 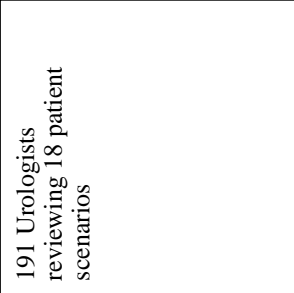 & 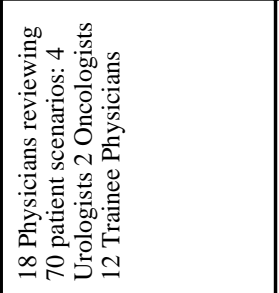 & 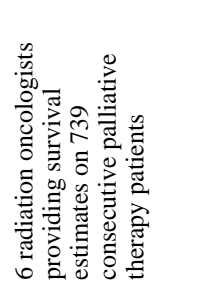 & 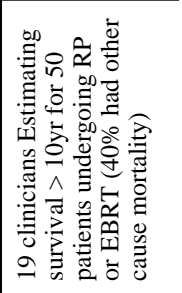 \\
\hline 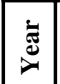 & $\stackrel{2}{2}$ & $\hat{\sigma}$ & ठิ & ڤ్ & ڤ్ & 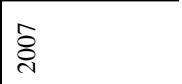 \\
\hline 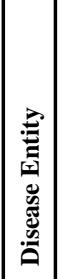 & 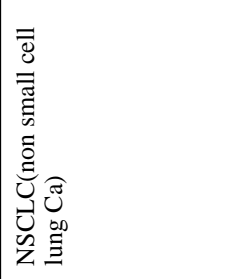 & 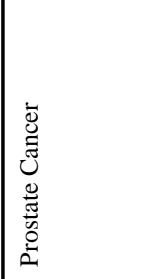 & 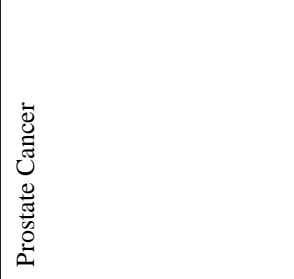 & 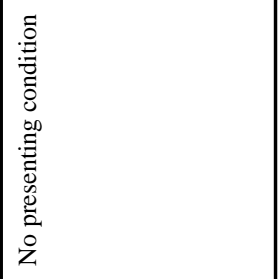 & 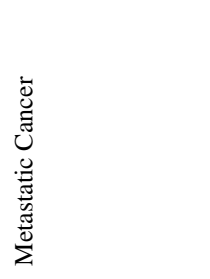 & 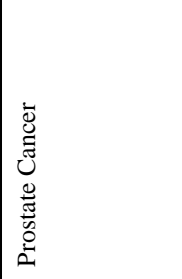 \\
\hline 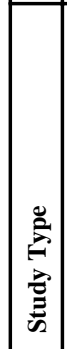 & 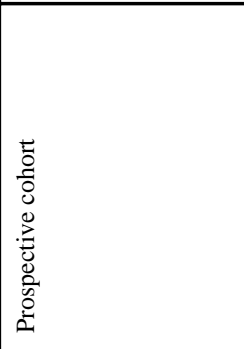 & 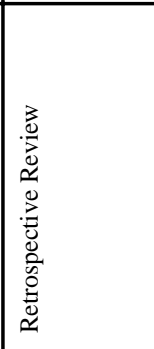 & $\sum_{\grave{D}}^{\grave{D}}$ & 害 & 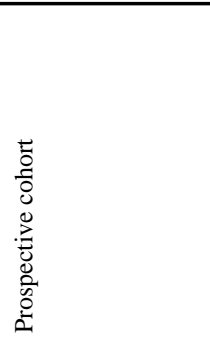 & 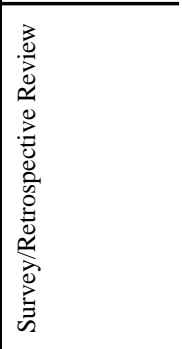 \\
\hline 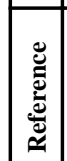 & 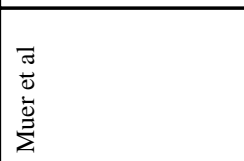 & 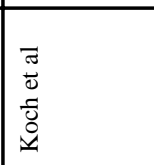 & 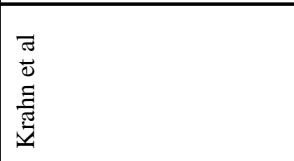 & $\mid \begin{array}{l}\pi \\
\frac{\pi}{0} \\
0 \\
0 \\
00 \\
3 \\
3\end{array}$ & $\begin{array}{l}\overline{0} \\
\overline{0} \\
0 \\
0 \\
0 \\
\text { U }\end{array}$ & \begin{tabular}{|l}
$\dot{\pi}$ \\
$\tilde{0}$ \\
$\tilde{0}$ \\
$\tilde{N}$ \\
3
\end{tabular} \\
\hline
\end{tabular}

Eur Urol. Author manuscript; available in PMC 2015 November 01. 


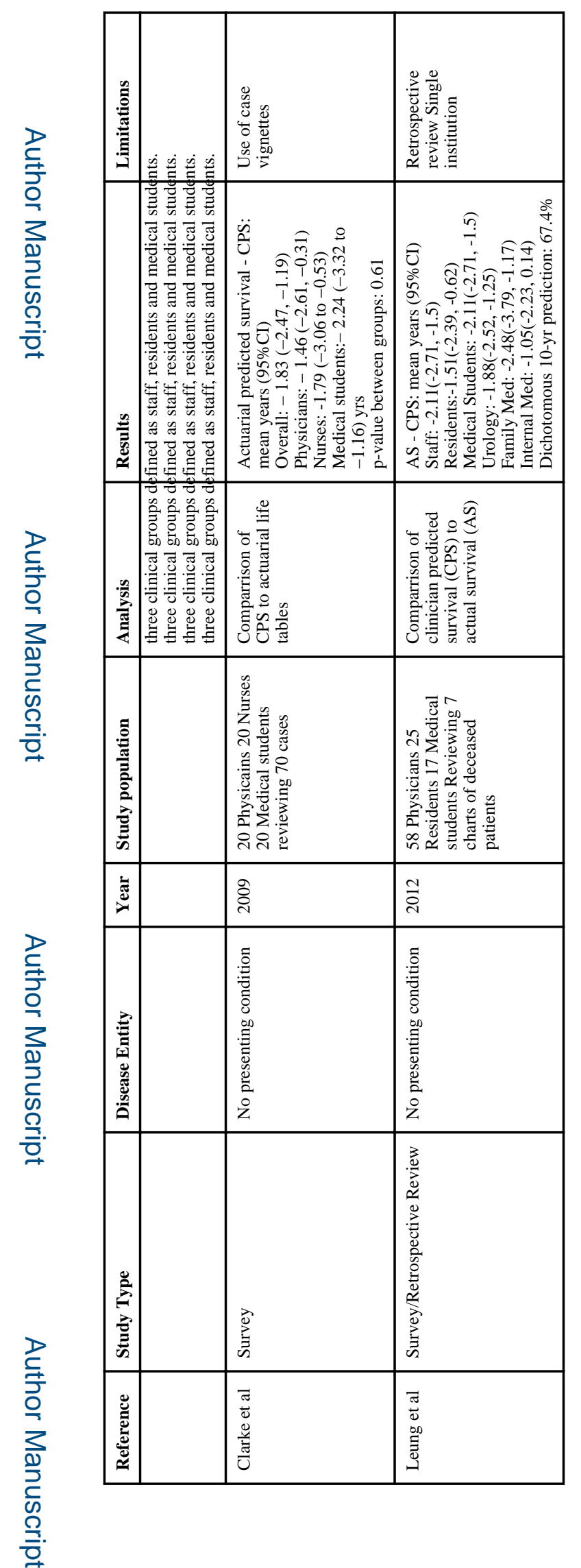

Eur Urol. Author manuscript; available in PMC 2015 November 01. 


\begin{tabular}{|c|c|c|c|c|c|}
\hline ) & 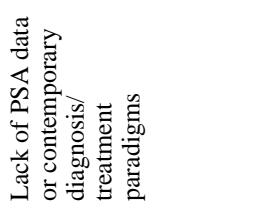 & 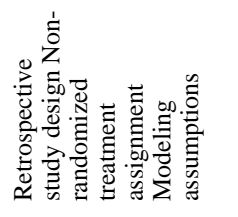 & 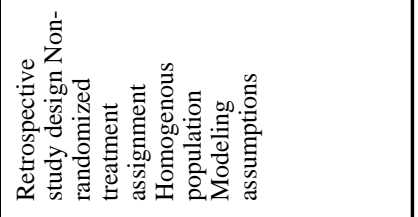 & 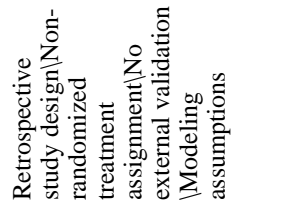 & 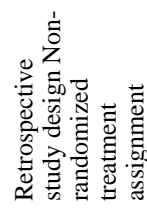 \\
\hline 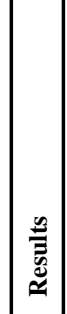 & 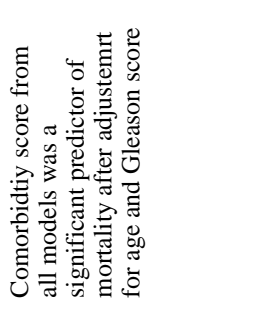 & 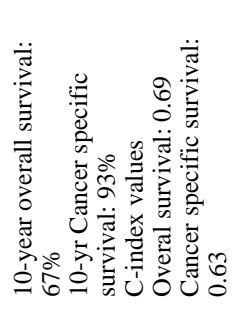 & 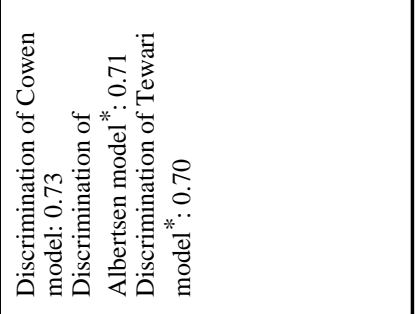 & 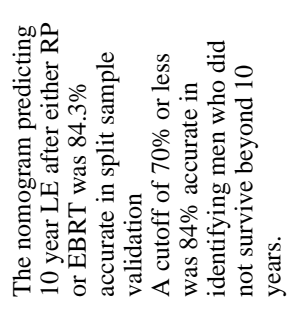 & 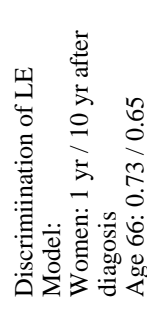 \\
\hline 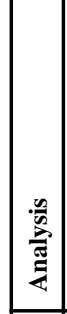 & 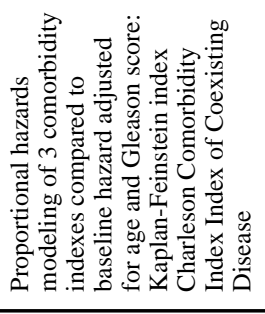 & 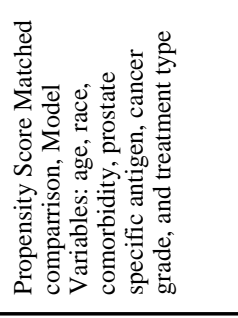 & 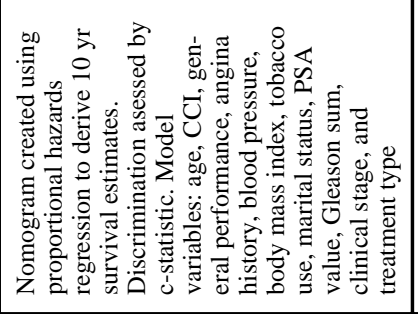 & 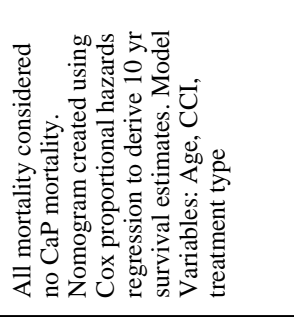 & 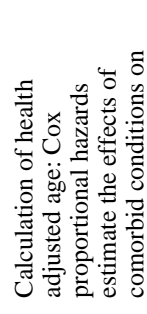 \\
\hline 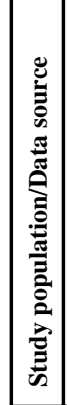 & 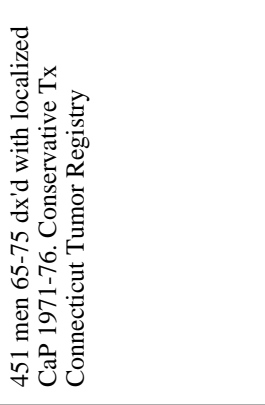 & 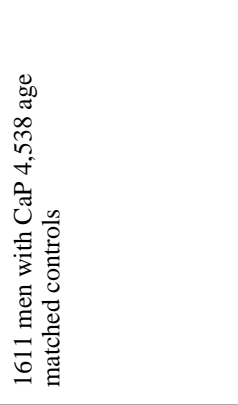 & 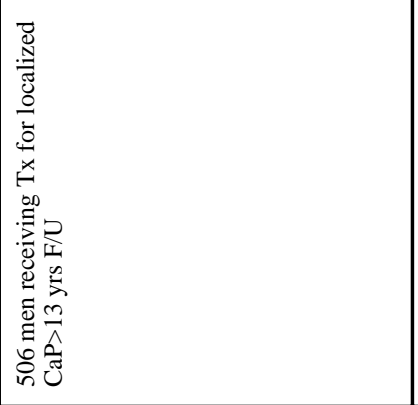 & 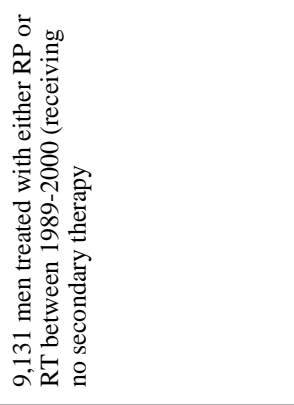 & 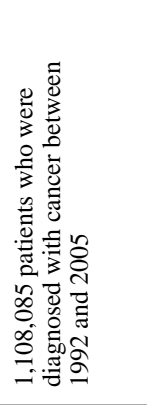 \\
\hline$\stackrel{\Xi}{\mathbb{\Xi}}$ & $\stackrel{\circ}{\circ}$ & 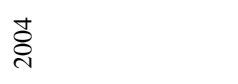 & 苂 & 亏્సે & $\stackrel{m}{\vec{i}}$ \\
\hline 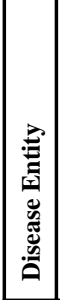 & 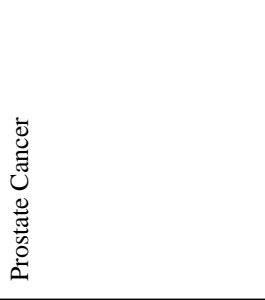 & 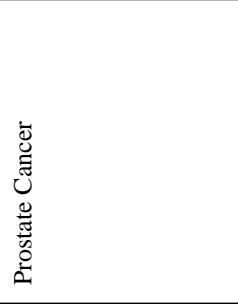 & 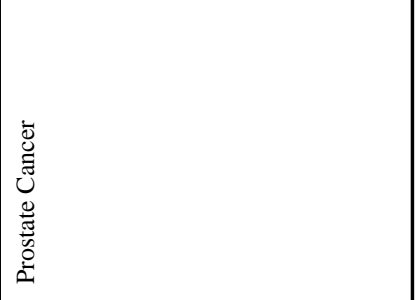 & 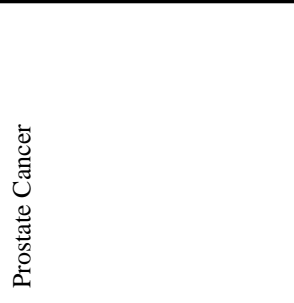 & 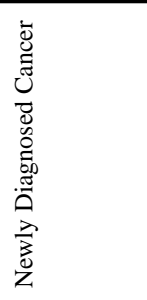 \\
\hline 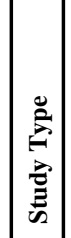 & 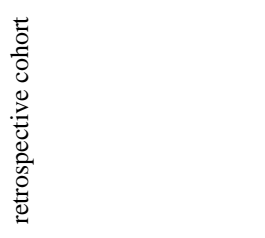 & 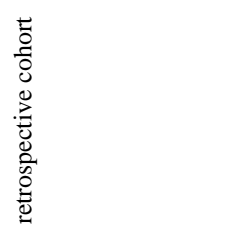 & 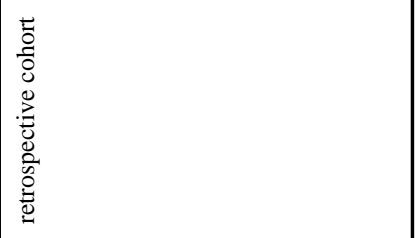 & 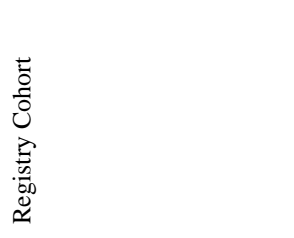 & 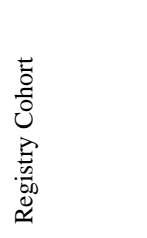 \\
\hline 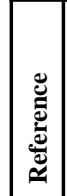 & 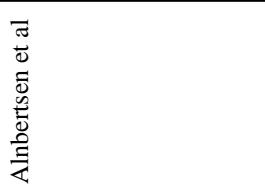 & 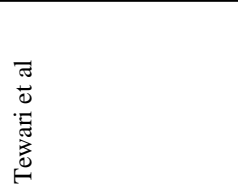 & $\begin{array}{l}\bar{J} \\
\tilde{0} \\
\tilde{\Xi} \\
\bar{\Xi} \\
0\end{array}$ & 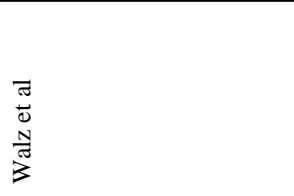 & 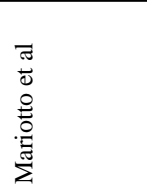 \\
\hline
\end{tabular}

Eur Urol. Author manuscript; available in PMC 2015 November 01. 


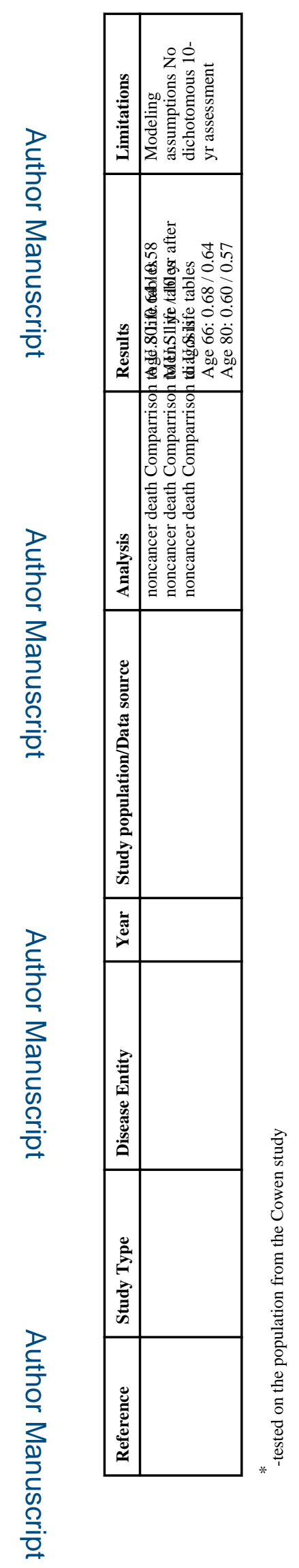

